\title{
Kelly GREENHILL, Weapons of Mass Migration: Forced Displacement, Coercion, and Foreign Policy
}

\section{Marc-Antoine Pérouse de Montclos}

\section{(2) OpenEdition}

Édition électronique

URL : https://journals.openedition.org/remi/5859

DOI : $10.4000 /$ remi.5859

ISSN : $1777-5418$

Éditeur

Université de Poitiers

\section{Édition imprimée}

Date de publication : 1 juin 2012

Pagination : 173-174

ISBN : 979-10-90426-04-7

ISSN : 0765-0752

Référence électronique

Marc-Antoine Pérouse de Montclos, « Kelly GREENHILL, Weapons of Mass Migration: Forced Displacement, Coercion, and Foreign Policy", Revue européenne des migrations internationales [En ligne], vol. 28 - n² | 2012, mis en ligne le 10 octobre 2012, consulté le 16 avril 2022. URL : http:// journals.openedition.org/remi/5859 ; DOI : https://doi.org/10.4000/remi.5859 


\section{NOTES DE LECTURE}

\section{BONNET, Doris}

Repenser l'Hérédité. - Paris : Archives contemporaines, 2009. - $116 \mathrm{p}$. ISBN : 978-2-914610-97-1

$D$ oris Bonnet, anthropologue, africaniste, directrice de recherches à l'IRD, analyse dans un livre court et dense, les multiples enjeux sociaux que soulève une maladie génétique, la drépanocytose, qui atteint en France en majorité des populations originaires des Antilles et d'Afrique sub-saharienne. À travers l'étude des représentations scientifiques et ordinaires de l'hérédité, des pratiques et discours recueillis dans un service hospitalier et auprès des familles, l'auteur touche à des questions relatives aux identités, aux stéréotypes raciaux et culturels, mais aussi aux stratégies que les familles, notamment les femmes, adoptent face aux injonctions, douloureuses et contradictoires, dans lesquelles elles se trouvent.

Un premier chapitre interroge la construction de cette maladie comme catégorie racialisée et ethnicisée : l'auteur montre, tout d'abord, comment les milieux médicaux ont élaboré - souvent à partir d'études en sciences sociales - des catégories d'ethnies figées qu'ils mobilisent pour justifier la non observance des originaires d'Afrique de l'Ouest, populations considérées comme les moins instruites et les plus rurales ${ }^{l}$. Or le référent ethnique est partout "un signifiant flottant $\|^{2}$ et en l'occurrence peu pertinent pour les populations rencontrées dans le cadre de cette enquête qui se définissent autrement, soit parce que la famille dispose de plusieurs origines, soit parce que les personnes

1 On peut aussi se référer au discours du 26 juillet 2007, à Dakar au Sénégal, de Nicolas Sarkozy véhiculant les mêmes stéréotypes d'archaïsmes des populations africaines et à la réponse des historiens africains et africanistes, cf. BA KONARÉ Adame (Dir.) (2009) Petit précis de remise à niveau de l'histoire africaine à l'usage du Président Sarkozy, Paris, La Découverte, $347 p$.

2 Jean-Loup Amselle, cité par Bonnet (p. 17). Cf. AMSELLE Jean-Loup (1985) Éthnies et espaces : pour une anthropologie topologique, in Jean-Loup Amselle et Elikia M'Bokolo, $\mathrm{Au}$ cœur de l'ethnie. Ethnie, tribalisme et État en Afrique, Paris, La Découverte, p. 37. ne connaissent pas l'ethnie de leurs parents (notamment les jeunes immigrés ou enfants d'immigrés), soit parce que leur urbanisation au pays d'origine ou leur migration rend obsolète une identification " ethnique ».

À cet imaginaire ethnique se superpose la référence raciale. Car malgré la réfutation de la scientificité de la notion de "race » concernant les êtres humains, l'épidémiologie (notamment nord-américaine, mais pas seulement) continue de l'utiliser en réifiant la drépanocytose comme maladie des "Noirs" ou des Africains alors qu'elle touche aussi des populations méditerranéennes et asiatiques. De même, alors qu'il est reconnu aujourd'hui que les gènes ont toujours circulé et que la fréquence d'un gène est le résultat des migrations et non le marqueur d'une "race " ou d'un isolat, le discours stigmatise les mariages au plus proche-notamment dans la parenté - induisant en erreur des personnes qui se croient à l'abri de la transmission en prenant un conjoint géographiquement éloigné et sans lien de parenté.

Cette maladie génétique s'insère et reproduit ainsi un ensemble de stéréotypes sur les Africains résidant en France, construits dans une altérité indépassable. Elle devient aussi, par réflexivité pour certains groupes d'influence, un élément de revendication pour une discrimination positive sur la base " raciale" ou « ethnique ».

Le deuxième chapitre est consacré à la rencontre tumultueuse, qui se joue pendant le suivi de grossesse, entre les multiples représentations de ce qui fait un enfant. En effet, des imaginaires protéiformes de la procréation (mélange des eaux du père et de la mère dans les sociétés rurales d'Afrique de l'Ouest, fruit du souffle spermatique dans la tradition aristotélicienne européenne, héritage du sang paternel ou maternel dans les systèmes à descendance unilatérale, au contraire de l'un et de l'autre dans les systèmes indifférenciés) se confrontent avec la vision mendélienne 
savante des médecins et ordinaire des populations instruites. Cette confrontation crée des tensions entre les préconisations des médecins et les décisions des familles souvent soupçonnées d'incapacité à accéder au savoir savant. Or l'ethnologue démontre comment les patients comme les soignants sont enserrés dans des systèmes de représentations qui figent le regard qu'ils portent sur leur interlocuteur-trice et sur la maladie. Par exemple, les hommes refusent souvent de porter la culpabilité de la transmission de la maladie lorsqu'eux-mêmes ne sont pas malades, mais porteurs sains. La faute est reportée sur la femme, même si elle est porteuse saine, surtout si plusieurs de leurs enfants décèdent. Ce rejet dérive de rapports de genre inégalitaires en Afrique de l'Ouest qui rendent les femmes responsables de la stérilité, des décès ou des handicaps des enfants, et non de l'impossibilité d'accès au savoir génétique comme le supposent les soignants. Comment comprendre autrement que des femmes peu instruites acceptent, elles, leur implication dans la transmission? Les décisions dépendent aussi de l'expérience et des choix individuels. Certains hommes, ainsi, resserrent leurs liens avec leur épouse et leurs enfants malades. L'auteur dévoile donc que les soignants sont autant traversés par des représentations inégalitaires du genre que leurs patients africains.

La drépanocytose révèle et participe aussi aux transformations des structures familiales opérées en Afrique et en migration. En effet, la théorie mendélienne centre la filiation sur le noyau biologique "père, mère, enfant" et le détache de la parenté et du lignage. L'enfant devient un investissement plus personnalisé et le fort secret qui imprime cette maladie renforce le risque d'isoler le noyau familial de la parenté, et plus encore la mère et ses enfants, lorsque le père se désinvestit. D'un autre côté, et l'auteur $y$ revient dans le dernier chapitre, le système socio-sanitaire français permet aux femmes de prendre des décisions par elles-mêmes, de se distancier d'un système de pouvoir lignager et patriarcal. Dans cet entrelacs des pouvoirs, les femmes, selon leur histoire et leurs ressources, peuvent y trouver de quoi se construire en tant que sujet.
Le chapitre sur les dépistages néonatal et prénatal s'avère particulièrement éclairant pour l'anthropologue comme pour les soignants. Doris Bonnet démontre que les tensions et les incompréhensions entre familles migrantes et professionnels de santé proviennent moins d'une culture imaginée archaïque que des décalages entre les systèmes sanitaires et législatifs africains et français. Ainsi, le dépistage prénatal n'existe pas dans nombre des pays d'origine et n'est donc pas familier. Surtout, l'interruption médicale de grossesse (IMG) est interdite ou très restreinte. Or, les médecins français associent fréquemment, dans les consultations observées, dépistage et IMG, ce qui introduit une forte ambiguïté sur le dépistage perçu comme un premier pas vers l'interruption de grossesse. Le refus $d u$ dépistage n'est souvent pas compris par les médecins qui au final l'attribuent à la religion ou à la culture. Les femmes se réapproprient ces arguments en sachant qu'évoquer leur religion sera davantage accepté par le personnel de santé que leur désir d'enfant en dépit des risques encourus.

À travers le cas spécifique d'une maladie chronique à transmission génétique, cet ouvrage concentre une multitude de questions relatives aux représentations qu'on se fait des migrants, particulièrement des Africains, mais aussi aux profonds bouleversements familiaux et personnels qu'introduit le suivi socio-sanitaire d'une maladie. L'anthropologie que pratique Doris Bonnet se pose ainsi comme un éclairage serein et objectif de la relation thérapeutique avec des populations immigrées ou descendantes d'immigrés. Elle éclaire les tensions et ouvre des voies sur ce qui pourrait être dénoué lorsque les uns et les autres accepteront de voir en face d'eux des êtres humains, avec toute la complexité qu'ils/elles transportent par leur histoire de vie. L'auteur montre aussi que les dispositifs sanitaires français sont une opportunité pour les femmes africaines de s'autonomiser, mais qu'elles doivent s'affirmer pour ne pas tomber dans d'autres formes d'assignations portées par le pouvoir bio-médical. On peut regretter qu'un sous-titre n'explicite pas davantage le contenu de l'ouvrage ce qui attirerait un lectorat large, 
tels que les professionnels de santé, les spécialistes des migrations et du genre, qui devraient être particulièrement intéressés par cet essai.

Virginie Vinel Maître de conférences Université Paul Verlaine de Metz Laboratoire Lorrain des Sciences Sociales

\section{GREENHILL, Kelly}

Weapons of Mass Migration: Forced Displacement, Coercion, and Foreign Policy. - Ithaca, NY: Cornell University Press, 2010. - $342 \mathrm{p}$.

ISBN : 978-0-8014-4871-3

$$
L_{t}^{e}
$$

e livre de Kelly Greenhill constitue un travail important qui, bizarrement, est pour l'instant resté inaperçu en France. Dans une perspective politique, il traite en l'occurrence de l'instrumentalisation diplomatique de la pression migratoire par les États, du Sud comme du Nord. L'auteur s'avère particulièrement convaincant lorsqu'il nous invite à ne pas sous-estimer la capacité des pays en développement à contrôler et exploiter les migrations transfrontalières, y compris en cas de débordement.

En guise d'introduction, Kelly Greenhill analyse ainsi comment, en 2004-2006, la Libye de Mouammar Kadhafi a négocié avec Bruxelles la levée des dernières sanctions économiques et la reprise des programmes de coopération de l'Union européenne en menaçant d'ouvrir ses frontières et de laisser passer les migrants africains désireux d'entrer dans l'espace Schengen. À l'occasion, rappelle l'auteur, certains États ont d'ailleurs mis leur menace à exécution et procédé à des expulsions massives. Au moment de la première crise du Golfe et de l'invasion du Koweït par l'Irak en 1990, l'Arabie Saoudite, notamment, a chassé les immigrés yéménites afin d'inciter le gouvernement de Sanaa à revoir sa position, qui était favorable à Saddam Hussein. Bien entendu, le jeu des frontières s'est aussi exercé dans le sens contraire - celui de la fermeture - et le livre de Kelly Greenhill regorge d'exemples puisés parmi les régimes communistes qui, eux, avaient précisément essayé de bloquer les sorties de leur territoire, et non les entrées.

De ce point de vue, les cas d'études analysés en annexe s'avèrent être une précieuse mine d'informations. En effet, l'apport du livre de Kelly Greenhill se situe bien au niveau de l'étendue des connaissances, de la diversité des zones couvertes et de la variété des périodes traitées. L'auteur le dit lui-même. En soi, "la manipulation et l'exploitation politiques des migrations transfrontalières n'ont rien de nouveau et de particulièrement inhabituel. Elles puisent leurs racines dans une histoire longue, en temps de guerre comme de paix » (p. 262). De fait, l'instrumentalisation des flux de réfugiés par la rétention ou, au contraire, la création délibérée de crises migratoires, conjuguée à la menace d'exodes et d'expulsions, constitue un phénomène ancien et récurrent qui ne date nullement de la fin de la guerre froide. L'originalité de Kelly Greenhill est d'affirmer que le procédé se révèle plus efficace que les sanctions économiques, en particulier lorsqu'il vise des régimes démocratiques et donc plus sensibles à des pressions et des normes humanitaires. Depuis la signature de la Convention de Genève en 1951, l'auteur recense ainsi plus de cinquante cas de la sorte, dont plus de la moitié ont réussi à faire plier leur cible.

Deux enseignements majeurs ressortent d'une telle analyse. Le premier est que l'instrumentalisation des crises migratoires ne se restreint pas aux pays en développement et ne date pas de la fin de la guerre froide. En cela, l'ouvrage de Kelly Greenhill se distingue de la vulgate des théoriciens des nouvelles guerres qui se focalisent sur l'Afrique et redécouvrent avec effroi que les civils sont les premières victimes des conflits armés. Pour être juste, d'autres auteurs avaient déjà étudié de pareils phénomènes dans la première moitié du XXe siècle. Anita Prazmowska, par exemple, a montré comment, à partir de 1939, le gouvernement polonais en exil à Londres a essayé d'attirer ses ressortissants fuyant l'occupation nazie afin 
de les recruter dans les troupes alliées ${ }^{3}$. Mais Kelly Greenhill est le premier à rassembler un grand nombre de cas d'études de manière aussi cohérente et démonstrative.

Or il ressort de son livre un autre point saillant, presque systématique, à savoir le rôle structurant de l'aide internationale dans l'instrumentalisation et la gestion des crises migratoires. Au-delà de ses aspects économiques ou humanitaires, la demande d'assistance est parfois de nature militaire, à l'instar du président en exil Jean-Bertrand Aristide qui, en 1992-1994, avait évoqué la menace d'un exode de boat people haïtiens vers les côtes de Floride pour inciter les États-Unis à renverser la junte au pouvoir à Port-au-Prince. Kelly Greenhill nous invite ainsi à reconsidérer l'expulsion des Indiens d'Ouganda en 1972. À l'époque, il paraissait évident qu'Idi Amin Dada voulait mettre la main sur les propriétés d'une communauté réputée pour son aisance matérielle. Mais une bonne partie des Indiens visés disposaient aussi de la nationalité britannique et leur renvoi visait également à convaincre la GrandeBretagne de reprendre son aide militaire, qu'elle venait de réduire du fait des errements de la dictature au pouvoir à Kampala.

Bien entendu, le chantage à l'aide par le moyen de la pression migratoire ne s'est pas limité à une négociation entre le Nord et le Sud. En 1984, par exemple, le président de l'Allemagne de l'Est, Erich Honecker, a menacé l'Ouest de laisser passer les demandeurs d'asile en provenance d'Asie ou du Moyen-Orient. Il a alors obtenu du gouvernement de Bonn une aide économique en échange de sa "bonne» volonté, qui consistait en fait à maintenir l'étanchéité du Mur de Berlin! L'initiative, il est vrai, a également pu venir des puissances occidentales dans une logique qui relevait moins $d u$ chantage que d'une inquiétude soigneusement entretenue par les pays potentiellement bénéficiaires. En 1990 puis 1997, c'est ainsi l'Italie qui a décidé d'augmenter son aide à l'Albanie

3 PRAZMOWSKA Anita (1988) Polish Refugees as Military Potential: Policy Objectives of the Polish Government in Exile, in Anna Bramwell (Ed.), Refugees in the age of total war, London, Unwin Hyman, pp. 219-232. pour essayer de contenir l'arrivée des boat people qui traversaient l'Adriatique sur des embarcations de fortune. Aujourd'hui, les pays $d u$ Sud sont tout aussi concernés, à l'instar de la Chine qui, depuis le milieu des années 1990, maintient la Corée du Nord sous perfusion afin d'éviter un afflux de réfugiés et de miséreux sur son territoire.

De là à imaginer que les migrants ou les candidats au départ soient toujours les otages d'un vaste jeu diplomatique qui les dépasse, il $y$ a certes un pas qu'on ne saurait franchir. De ce point de vue, Kelly Greenhill aurait sûrement gagné à intégrer davantage dans son analyse le rôle politique des migrants eux-mêmes. Il n'en reste pas moins que son ouvrage est hautement recommandable.

Marc-Antoine Pérouse de Montclos CEPED (Centre Population \& Développement) UMR196 Paris Descartes - INED - IRD 